\title{
Withaferin A inhibits cell proliferation of U266B1 and IM-9 human myeloma cells by inducing intrinsic apoptosis
}

\author{
Lan Li, Ben Niu, Weihua Zhang, Limin-Hou and Yan Zheng ${ }^{\otimes}$ \\ Department of hematopathology, Shaanxi Provincial People's Hospital, Xi 'an, Shaanxi, China
}

\begin{abstract}
Withaferin A, a withanolide obtained from Withania somnifera exhibits remarkable pharmacological properties. Withaferin A has been reported to exert cytotoxic effects against human multiple myeloma cells. Nevertheless, the in-depth understanding of the withaferin $A$ induced antiproliferative effects against human myeloma cells is still unclear. The results showed that withaferin $A$ inhibited the viability of six different myeloma cells with a lowest $\mathrm{IC}_{50}$ of $9 \mu \mathrm{M}$ against the U266B1 and IM-9 cell lines. Withaferin $A$ inhibited the viability and colony formation of the U266B1 and IM-9 cells in a dose and timedependent manner. The DAPI and annexin V/PI staining assays revealed that withaferin $A$ exerts anticancer effects against the human myeloma cells via induction of apoptosis. The induction of apoptosis in U266B1 and IM-9 cells was associated with upregulation of Bax and cytochrome $c$, downregulation of $\mathrm{Bcl}-2$ and activation of PARP, caspase-3 and capase-9 cleavage. Additionally, withaferin A triggered the production of ROS in human myeloma cells indicative of ROS mediated apoptosis in human myeloma cells. The treatment of the U266B1 and IM-9 with ascorbic acid (antioxidant) could prevent the withaferin A mediated ROS production and the withaferin $A$ induced antiproliferative effects. Collectively, the results show that withaferin $A$ inhibits human myeloma cell proliferation via ROS mediated intrinsic apoptosis.
\end{abstract}

Keywords: myeloma, withanolide, Withaferin A, apoptosis, anti-cancer

Received: 02 October, 2021; revised: 24 October, 2021; accepted: 27 October, 2021; available on-line: 02 March, 2022

凶e-mail: 280467792@163.com

Abbreviations: ROS, reactive oxygen species; DAPI, 4,6-diamidino2-phenylindole; CCK-8, cell counting kit-8; DMSO, dimethyl sulfoxide

\section{INTRODUCTION}

Multiple myeloma or myeloma is a malignant neoplastic disorder of blood plasma cells (Muccio et al., 2016). It is characterized by the uncontrolled growth and accumulation of plasma cells in bone marrow (Szudy-Szczyrek et al., 2016). The most frequent clinical symptoms associated with multiple myeloma include, but are not limited to, kidney injury, bone destruction and paraproteinemia (Kumar \& Rajkumar, 2018). Myeloma is ranked as the second most common hematologic disorder and accounts for $10 \%$ of all the malignant blood disorders (Dicato, 2018). Besides, it makes up to 1 percent of all human cancers at global level (Kazandjian, 2016). Despite the great progress in the therapeutic regimes over few decades, the multiple myeloma is still incurable because of the molecular heterogeneity of malignant plasma cells which is responsible for the frequent disease recurrence within noticeably short periods of diminution (Munshi \& Avet-Loiseau, 2011). Recent years have witnessed a paradigm shift in the management of human myeloma. There is growing support that before assigning a particular treatment procedure for myeloma, the disease development and subsequent progression must be intensively explored for achieving better clinical results (Kumar, 2019). Moreover, the recent studies have envisaged how to target specific genetic abnormalities to avoid the treatment failures arising as a result of instances of development of drug resistance (Pinto et al., 2020). In connection with this, there is an urgent need of examining the efficacy of natural products for development of novel drugs so as to enable ourselves to simulate a judicious drug combination against human myeloma in the future (Kumar, 2019). With the same objective, the anti-cancer effects of Withaferin A were investigated against human myeloma cells in the present study. Withaferin A is the most bio-active withanolide, highly oxygenated lactone from Withania somnifera known for antioxidant, anti-inflammatory, and anti-depressant properties (Singh et al., 2011; Berghe et al., 2012). The effectiveness of Withaferin A to serve as anti-cancer agent has been assessed against different types of human cancer cells including breast cancer, colon cancer, head and neck cancer, leukemia, ovarian cancer, multiple myeloma to name a few (Hassannia et al., 2020). Withaferin has been shown to inhibit the proliferation of the human multiple and induce their cell differentiation (Issa \& Cuendet, 2017). However, the in-depth underlying mechanism for the withaferin A induced antiproliferative effects against the human myeloma cells has not been explored. This study for the first-time reports that the anticancer effects of withaferin A against the human myeloma cells is due to ROS mediated intrinsic apoptosis.

\section{MATERIALS AND METHODS}

\section{Cell lines and culture conditions}

The six multiple myeloma cell lines (MOLP-8, U266B1, RPMI-8226, KMS-11, IM-9 and JJN-3) were purchased from American Type Culture Collection (ATCC, USA). The myeloma cell lines were cultured using Roswell Park Memorial Institute medium (RPMI1640, Gibco; Ireland). The culture medium was supplemented with 10\% fetal bovine serum (FBS, Gibco; Ireland) and streptomycin $(100 \mu \mathrm{g} / \mathrm{mL})-1 \%$ penicillin (100 $\mathrm{U} / \mathrm{mL}$ ). The myeloma cells were maintained in a humidified atmosphere at $37^{\circ} \mathrm{C}$ with $5 \% \mathrm{CO}_{2}$. Normal plasma cells (NPCs) were extracted form peripheral blood smear 
and cultured according to previously reported methods (Adham et al., 2020).

\section{Cell proliferation assay}

After treatment with varying doses for $24 \mathrm{~h}$ or varying durations with withaferin A in 96-well plates, the cell proliferation of myeloma and normal plasma cells was analyzed using the cell counting kit-8 (CCK-8, Beyotime Institute of Biotechnology) assay. Herein, immediately after treating with Withaferin A, the cells $\left(2 \times 10^{3} /\right.$ well $)$ were supplemented with $10 \mu \mathrm{L}$ CCK- 8 reagent. Cells were again incubated for $2.5 \mathrm{~h}$ at $37^{\circ} \mathrm{C}$. Using a microplate reader (Bio-Rad Laboratories, Inc.), the absorbance was measured at $450 \mathrm{~nm}$ to determine the cell proliferation.

\section{Colony formation assay}

For the analysis of colony formation, a total of $10^{5}$ U266B1 or IM-9 cells were grown for 14 days in 6 -well plates after being administered with varying Withaferin A doses for $24 \mathrm{~h}$ or with $9 \mu \mathrm{M}$ Withaferin A for $0,12,24,48$ or $96 \mathrm{~h}$ at $37^{\circ} \mathrm{C}$. After two weeks when the colonies were visible, the supernatant was removed, and the colonies formed were washed thrice with phosphatebuffered saline (PBS). Then, the colonies were fixed with $70 \%$ ethanol for $25 \mathrm{~min}$ and subsequently stained using $0.1 \%$ crystal violet for $20 \mathrm{~min}$. Photographs were taken with a camera and the Image J software (National Institutes of Health, USA) was used for estimating the number of colonies from each well and then percent colony formation was calculated.

\section{Analysis of cell apoptosis}

The U266B1 or IM-9 myeloma cells were incubated with $0,4.5,9.0$ or $18 \mu \mathrm{M}$ of Withaferin A for $24 \mathrm{~h}$ at $37^{\circ} \mathrm{C}$. Apoptotic signs were visualized using DAPI staining. Here, the variedly treated cells along with the untreated U266B1 or IM-9 cells were PBS washed and then fixed with $70 \%$ ethanol for $20 \mathrm{~min}$ at room temperature. The cells were then permeabilized with Triton X-100 (0.1\% in PBS) and stained with DAPI $(1 \mu \mathrm{g} / \mathrm{mL}$ in PBS) for $10 \mathrm{~min}$ in the dark at $37^{\circ} \mathrm{C}$. Finally, the nuclear morphology was studied using the fluorescence microscope.

The apoptosis of U266B1 or IM-9 myeloma cells was also assessed using Annexin V-FITC/PI staining method. Briefly, post-treatment; the cells were collected through centrifugation. After trypsinization, the cells were re-suspended in $185 \mu \mathrm{L}$ binding buffer and then supplemented with $5 \mu \mathrm{L}$ Annexin V-FITC and $10 \mu \mathrm{L}$ PI. This was followed by a dark incubation of $25 \mathrm{~min}$ at room temperature. Finally, the apoptosis of myeloma cells was measured using flow cytometry.

\section{Western blotting}

The U266B1 or IM-9 myeloma cells were treated with different doses of withaferin A for $24 \mathrm{~h}$ at $37^{\circ} \mathrm{C}$. Afterwards, the cells were harvested, and PBS washed. Total proteins were extracted from the myeloma cells using M-PERR Mammalian Protein Extraction Reagent and protease inhibitor (1:100). The Nano-Drop1000 spectrophotometer (Thermo Fisher Scientific) was used for the determination of total protein concentration. Then, $40 \mu \mathrm{g}$ of total protein were resolved by SDS-PAGE. The PAGE-gels were blotted to the PVDF membranes. $5 \%$ BSA/TBS-T was used for blocking the membranes. The membranes were incubated with primary antibodies against desired proteins overnight. The human $\beta$-actin served as an internal reference protein. This was followed by the incubation of PVDF membranes with antimouse IgG HRP-linked secondary antibodies. Specific protein bands were detected by a colorimetric reaction using Luminata TM Classico Western HP.

\section{Estimation of intracellular ROS-levels}

The intracellular ROS levels from differentially treated U266B1 or IM-9 myeloma cells were quantified using H2DCFH-DA. The ROS oxidize the cell permeable non-fluorescent dye H2DCFH-DA to a highly fluorescent (green) 2',7'-dichlorofluorescein (DCF). The myeloma cells pre-incubated with $2 \mu \mathrm{M}$ H2DCFH-DA for 35 min. The cells were then administered with different withaferin A doses or DMSO (negative control) for $2 \mathrm{~h}$. Post-treatment, the cells were harvested, PBS washed and then subjected to fluorescent microscopy. The relative ROS levels were determined using fluorescent spectrophotometer.

\section{Transwell migration and invasion assays}

The myeloma cell migration and invasion were analyzed using the transwell chambers either uncoated or Matrigel (BD Biosciences, USA) coated according to the manufacturer's instruction. For migration assay, U266B1 or IM-9 cells variably treated with withaferin A were grown in $150 \mu \mathrm{L}$ serum-free RPMI-1640 after treatment for $48 \mathrm{~h}$ at $37^{\circ} \mathrm{C}$. The cells were placed into a fibronectin-coated polycarbonate membrane inserts of a transwell apparatus. Culture medium alone was added into the lower chamber. Following $24 \mathrm{~h}$ incubation at $37^{\circ} \mathrm{C}$, the cells from the upper surface of the membrane were swabbed away with cotton while those adhering to its lower surface were fixed with $4 \%$ paraformaldehyde and then stained with $0.1 \%$ crystal violet solution for $25 \mathrm{~min}$. The invasion of myeloma cells was also analyzed through similar procedure except that the transwell membranes were coated in advance with $30 \mathrm{mg} / \mathrm{mL}$ Matrigel.

\section{Statistical analysis}

Each experiment was performed using three replicates and final results were shown as mean \pm standard deviation (S.D.). All the statistical analyses were carried out using GraphPad Prism 7.0 software (GraphPad Software, San Diego, CA, USA). The Student's $t$-test was performed to assess differences between two treatment groups. The $p$ values $<0.05$ were taken as the representative of statistically significant difference.

\section{RESULTS}

\section{Withaferin A inhibited in vitro proliferation and colony formation of myeloma cells}

Withaferin A, the most bio-active withanolide from Withania somnifera, is a highly oxygenated plant-based lactone (Figure 1A). A panel of six multiple myeloma cell lines (MOLP-8, U266B1, RPMI-8226, IM-9, KMS11 and JJN-3) and the normal plasma cells (PCs) were administered with varying concentrations of withaferin A to evaluate its effects on the myeloma cell proliferation. It was found that withaferin A inhibited the proliferation of all the myeloma cancer cell lines (Table 1). However, the growth of U266B1 and IM-9 myeloma cell line was most severely affected with an estimated $\mathrm{IC}_{50}$ of 
Table 1. Proliferation of different myeloma and normal plasma cells was determined by CCK-8 assay and presented as IC50 values

\begin{tabular}{lll}
\hline S. No. & Cell line & $\mathrm{IC}_{50}(\mu \mathrm{M})$ \\
\hline 1 & MOLP-8 & 18 \\
\hline 2 & U266B1 & 9 \\
\hline 3 & RPMI-8226, & 24 \\
\hline 4 & $\mathrm{IM}-9$ & 9 \\
\hline 5 & KMS-11 & 12 \\
\hline 6 & JJN-3 & 18 \\
\hline 7 & PCs & 74 \\
\hline
\end{tabular}

$9 \mu \mathrm{M}$ (Fig. 1B and 1C). Thus, U266B1 and IM-9 cells were used for further study. On the other hand, when the normal PCs were co-incubated with different doses of withaferin $\mathrm{A}$ for $24 \mathrm{~h}$ at $37^{\circ} \mathrm{C}$, the decline in cell pro- liferation was not very prominent and evident from the $\mathrm{IC}_{50}$ of $74 \mu \mathrm{M}$ that was observed for the normal plasma cells (Fig. 1D). Furthermore, the U266B1 and IM-9 myeloma cell growth was inhibited in time-dependent manner when the cancer cells were administered with $9 \mu \mathrm{M}$ withaferin A for varying incubation periods (Fig. 1E). Withaferin A also decreased the colony formation from U266B1 and IM-9 cancer cells in a dose-dependent manner (Fig. 1E). Collectively, the results signify the antiproliferative action of withaferin $\mathrm{A}$ against the human myeloma cells without much effect on the normal cell growth.

\section{Withaferin A induced apoptosis in myeloma cells}

To look for the possible mechanism for the cytotoxic action of withaferin A, U266B1 or IM-9 myeloma cells were treated for $24 \mathrm{~h}$ with $0,4.5,9.0$ or $18.0 \mu \mathrm{M}$ of withaferin A. The differently treated myeloma cells were DAPI stained, and it was observed that the with-

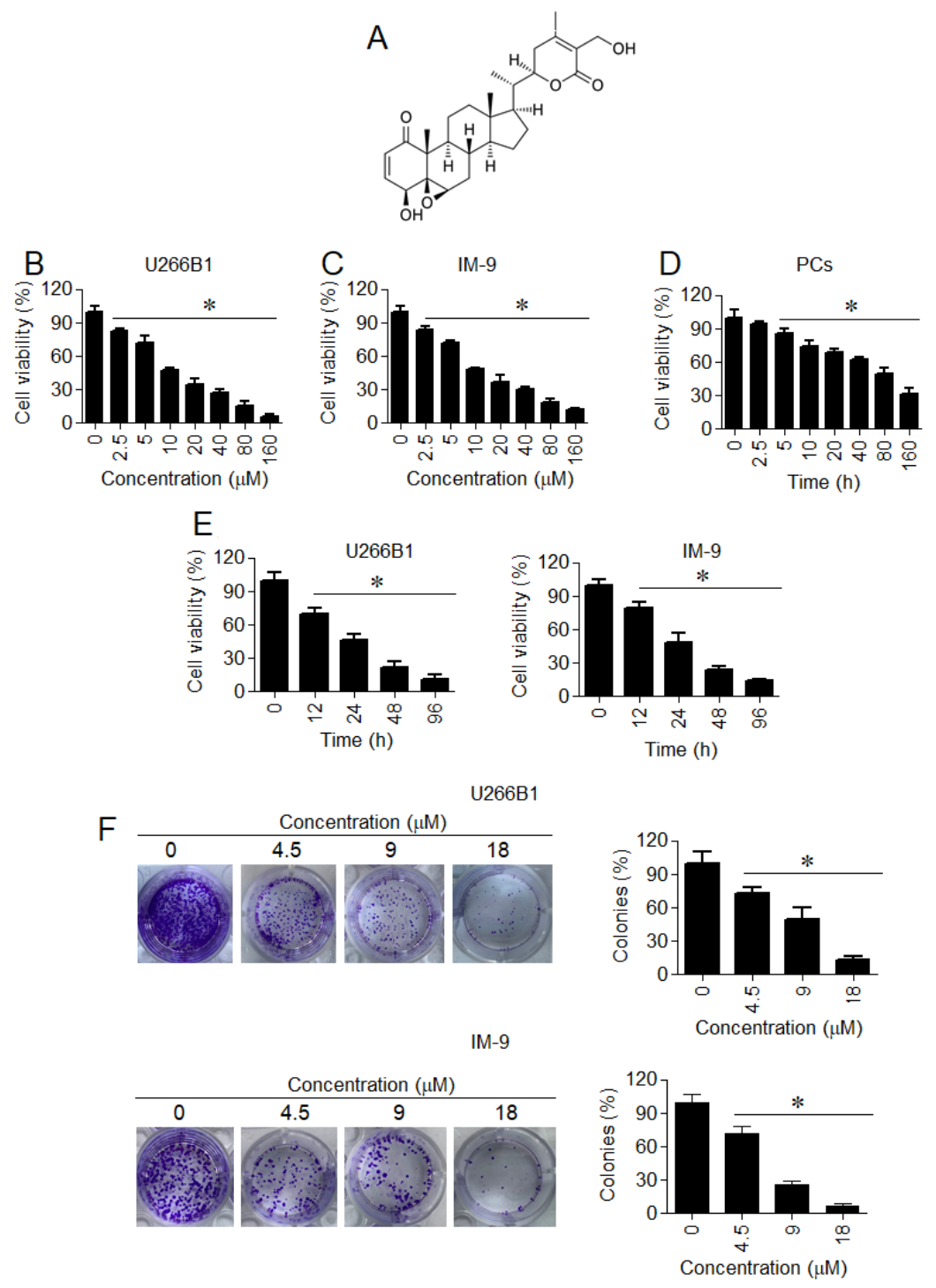

Figure 1. Withaferin inhibits in vitro myeloma cell proliferation.

(A) Chemical structure of Withaferin A (B) analysis of effects of withaferin A on proliferation of U266B1 myeloma cells by CCK-8 assay (C) analysis of effects of withaferin A on proliferation of IM-9 myeloma cells by CCK-8 assay (D) analysis of effects of withaferin A on proliferation of normal PCs by CCK-8 assay (E) analysis of effect of withaferin A administration for different durations on proliferation of U266B1 or IM-9 myeloma cells by CCK-8 assay (E) assessment of colony formation of U266B1 or IM-9 myeloma cells pre-administered with different doses of withaferin A. Experiments were performed in triplicates and presented as mean \pm S.D. $\left({ }^{*} P<0.05\right)$. 

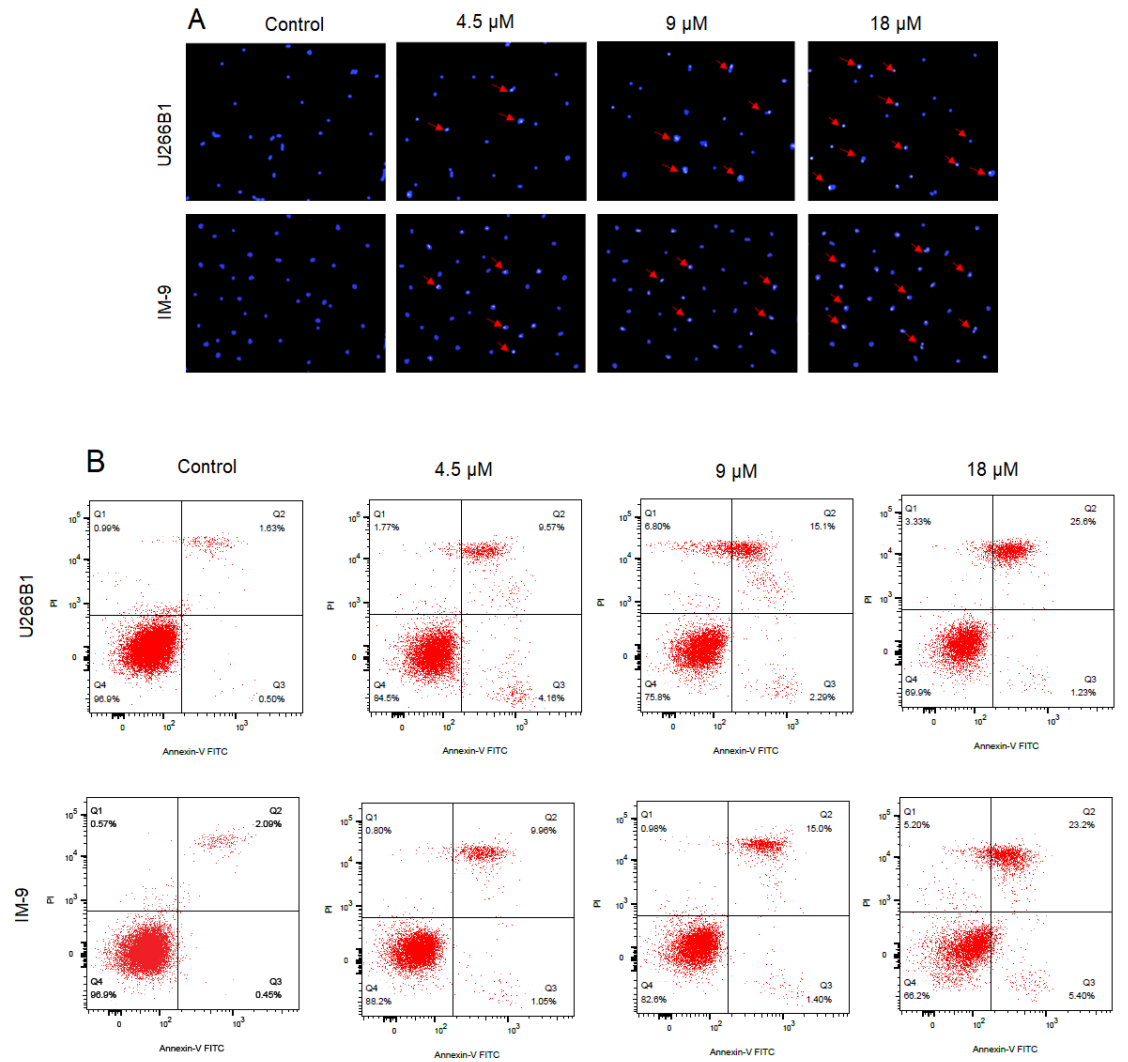

U266B1

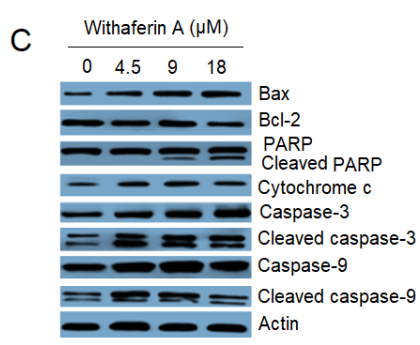

IM-9

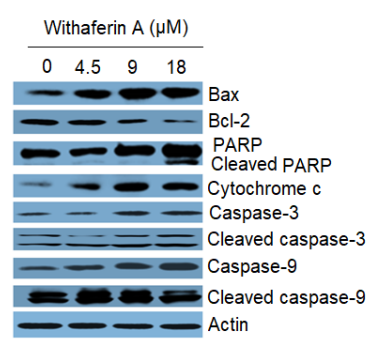

Figure 2. Withaferin induces apoptosis in myeloma cells.

(A) DAPI staining for the analysis of nuclear morphology of U266B1 or IM-9 cancer cells administered with varied concentrations of Withaferin A (B) Annexin V-FITC/PI staining for the analysis of apoptosis of U266B1 or IM-9 cancer cells administered with varied concentrations of Withaferin A (C) western blotting of apoptosis marker proteins from U266B1 or IM-9 cells administered with varied concentrations of Withaferin A. Experiments were performed in triplicates.

aferin A treated U266B1 or IM-9 myeloma cells exhibited signs of apoptosis evidenced by nuclear disintegration when analyzed by fluorescence microscopy and the signs were very prominent at higher treatment concentration (Fig. 2A). Annexin V-FITC/PI staining was used for further confirmation. The flow cytometry results indicated that the percentage of apoptotic myeloma cells increased with the increase in Withaferin A concentration (Fig. 2B). The percentage apoptotic U266B1cells increased from 2.13 in control to 26.83 at $18 \mu \mathrm{M}$ withaferin A. Similarly, the percentage of IM-9 apoptotic cells increased from 2.53 to $28.6 \%$ at $18 \mu \mathrm{M}$ withaferin $\mathrm{A}$. The induction of apoptosis in myeloma cells was finally confirmed by western blotting of apoptosis marker proteins. The expression levels of Bax and Cytochrome $c$ proteins were increased while that of $\mathrm{Bcl}-2$ protein decreased with increasing Withaferin A treatment doses (Fig. 2C). Treat- ment of Withaferin A was found to trigger the cleavage of caspase 3, caspase 9 and PARP proteins. The expression of caspase 3 was slightly increased while PARP and caspase 9 expression levels remained unaffected. The results show that withaferin A induced apoptosis in myeloma cells to inhibit their growth, in vitro.

\section{Withaferin A increased ROS-levels in myeloma cells}

To assess the effect of Withaferin A on intracellular ROS generation, the U266B1 or IM-9 myeloma cells treated with $0,4.5,9.0$ or $18 \mu \mathrm{M}$ Withaferin A along with untreated cancer cells were probed with the nonfluorescent dye H2DCFH-DA, which when oxidized gets converted into green, fluorescent DCF. The intensity of green, fluorescent signal increased with the increasing withaferin A concentration, which showed 

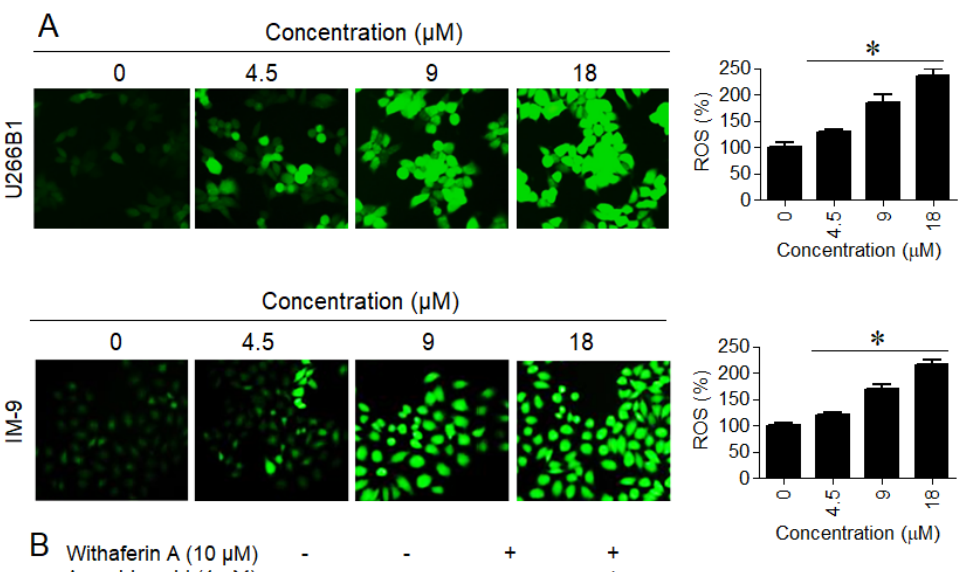
Withaferin $A(10 \mu \mathrm{M})$
Ascorbic acid $(1 \mu \mathrm{M})$
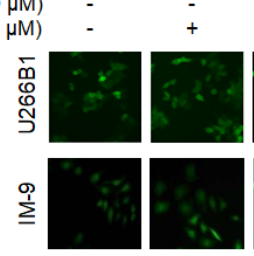

C
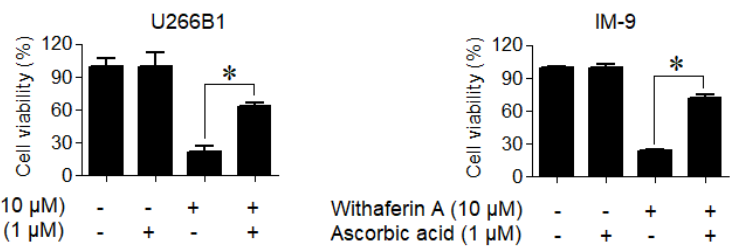

Figure 3. Withaferin A treatment led to ROS accumulation in myeloma cells.

(A) Fluorescence based analysis of ROS levels of U266B1 or IM-9 cancer cells treated with varied concentrations of Withaferin A (B) analysis of relative ROS generation in U266B1 or IM-9 myeloma cells administered with Withaferin A and/or ascorbic acid (C) analysis of relative percentage of viability of U266B1 or IM-9 myeloma cells administered with Withaferin A and/or ascorbic acid. Experiments were performed in triplicates and presented as mean \pm S.D. $\left({ }^{*} P<0.05\right)$.

that withaferin A augmented the generation of ROS in myeloma cells (Fig. 3A). The fluorescence intensity of DCF was used for estimation of the relative ROS levels of myeloma cells differentially treated with Withaferin A. The intracellular ROS levels were shown to be more than 2-fold higher at $18 \mu \mathrm{M}$ Withaferin A treatment (Fig. 3B). The administration of Withaferin A $(10 \mu \mathrm{M})$ treated U266B1 or IM-9 cancer cells with $1 \mu \mathrm{M}$ ascorbic acid, a ROS-scavenger, significantly declined the DCF fluorescence (Fig. 3C). Moreover, the ascorbic acid supplementation attenuated the anti-proliferative effects of withaferin A and enhanced the myeloma cell viability, considerably (Fig. 3D). The results thus indicate that withaferin A increased intracellular ROS generation and possibly induced apoptosis in myeloma cancer cells via this ROS accretion.

\section{Withaferin $\mathrm{A}$ abridged the migration and invasion of myeloma cells, in vitro}

The anti-cancer effects of withaferin A on U266B1 or IM-9 cells were also studied in terms of its effect on the cell migration and invasion, in vitro. Notably, the U266B1 or IM-9 myeloma cells were administered with exceptionally low concentrations of withaferin A (up to $8 \mu \mathrm{M})$ to over-rule its cytotoxic effects for studying their migration and invasion. The migration of myeloma cells was reduced in dose-dependent fashion (Fig. 4A). The percentage of cell migration was less than $30 \%$ at $8 \mu \mathrm{M}$ withaferin A concentration in comparison to the normal untreated myeloma cells. Withaferin A treatment simi- larly affected the cell invasion and the latter decreased by more than $70 \%$ when myeloma cells were administered with $8 \mu \mathrm{M}$ Withaferin A (Fig. 4B). Withaferin A might thus exhibit an anti-metastatic action against myeloma cells. Collectively, the results suggest that withaferin A induces ROS mediated apoptosis in myeloma cells (Fig. 5) and also inhibits cell migration and invasion.

\section{DISCUSSION}

Natural products are considered as a precious source for prevention and treatment of human ailments since times immemorial (Mousa, 2017). Cancer is still categorized as one of the dreadful clinical disorders threatening the human survival (Xia et al., 2017). A large number of naturally occurring chemical compounds have been shown to exhibit considerable therapeutic affects against different types of cancer cells (Cragg \& Pezzuto, 2016). Plant derived metabolites like taxol, vincristine, vinblastine etc. are some of the important chemotherapeutic agents presently used against human cancer (Iqba et al., 2017). Withaferin $A$ is the first plant-based anti-cancer withanolide, which was extracted way back in 1967 (Lee \& Choi, 2016). There are several reports that withaferin A exhibits anti-proliferative properties and inhibits the cancer cell growth both in vitro and in vivo (Kakar et al., 2017). Withaferin $A$ has been shown to exert its anticancer effects through the induction of apoptotic cell death and/or cell cycle arrest by targeting diverse molecular pathways (Tang et al., 2020). The present study 

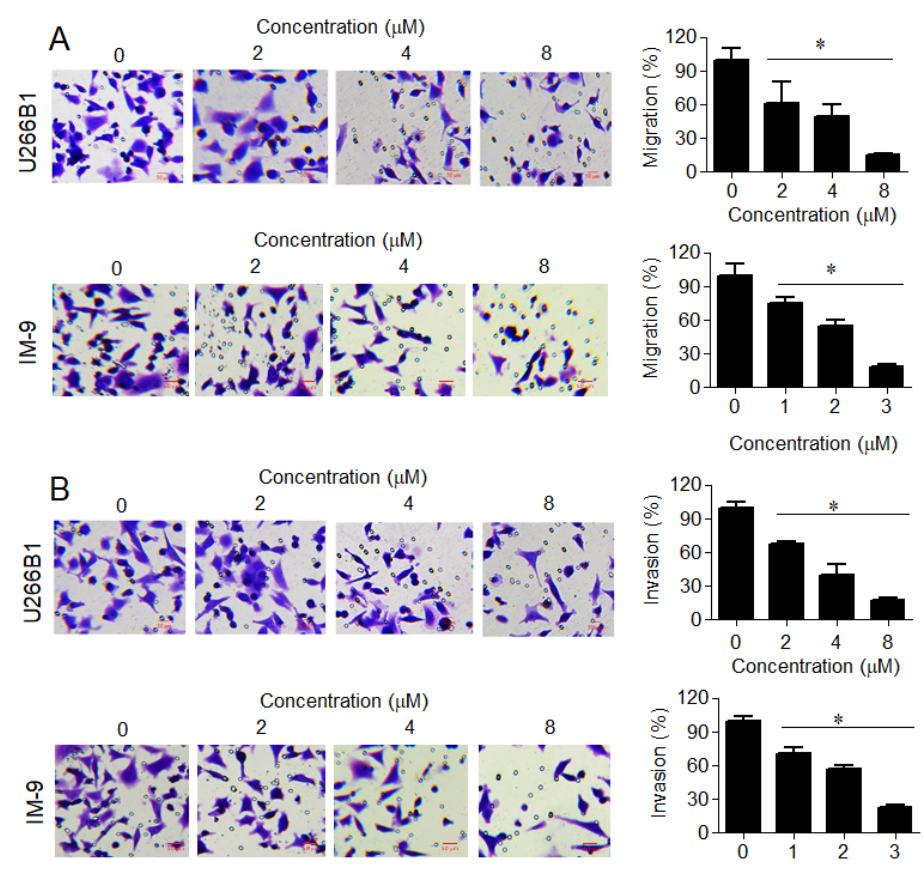

Figure 4. Withaferin A restrained migration and invasion of myeloma cells, in vitro

(A) Analysis of migration of U266B1 or IM-9 myeloma cells differentially administered with Withaferin A by transwell migration assay (B) analysis of invasion of U266B1 or IM-9myeloma cells differentially administered with Withaferin A by transwell invasion assay. Experiments were performed in triplicates and presented as mean \pm S.D. $\left({ }^{*} P<0.05\right)$.

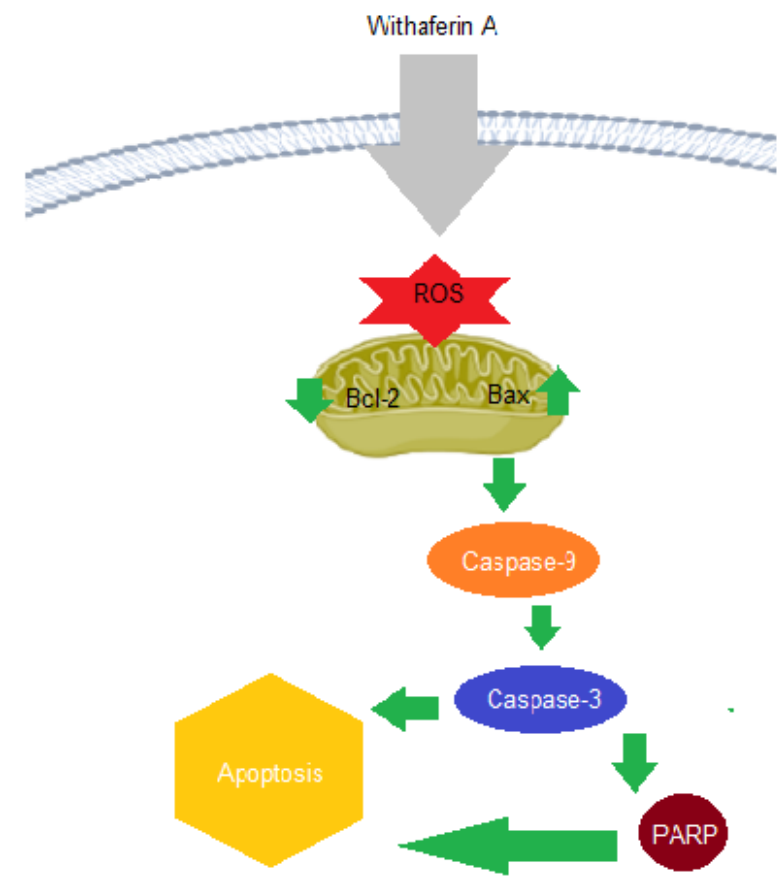

Figure 5. Model for the withaferin A induced apoptosis in myeloma cells.

Withaferin A triggers the accretion of ROS which triggers alteration of the apoptosis related proteins (Bcl-2, Bax, Caspase- 9 and -3 and PARP) eventually activating intrinsic apoptosis. connoted the dose-dependent and selective growth inhibitory property of withaferin A against the malignant plasma cells with little effect on the normal plasma cell viability, suggesting withaferin $A$ as a molecule of choice for drug design against the human myeloma (Ding \& Chen, 2018). Previously, the targeting of signal transducer and activator of transcription 3 (STAT3) was elucidated as the possible mechanism mediating the anti-cancer effects on the human myeloma cells via the apoptosis induction in vitro (Yco et al., 2014). The induction of apoptosis in myeloma cells was also confirmed by the results of the current study. Withaferin A triggered the activation of caspases that are otherwise present as inactive zymogens inside the cells (Shi, 2004). In addition, the apoptotic signal was modulated through Bax and Bcl-2 proteins (Naseri et al., 2015). Additionally, the myeloma cells exhibited higher cleaved PARP-1 levels, which have been shown to promote necrotic cell death (Eguchi et al., 1997). The caspase activation and subsequent PARP cleavage might be resulting from the release of mitochondrial cytochrome $c$ by withaferin A (Cai et al., 1998). The results of the present study suggested that the induction of apoptosis in myeloma cells was prompted via ROS-accretion. Similar mode of apoptosis induction has already been confirmed for withaferin against the human breast cancer cells (Hahm et al., 2010). ROS mediated induction of apoptosis in cancer cells is also true for other natural products and has been valued as potent anti-cancer strategy (Fulda, 2011). Withaferin A has been proven to have an anti-metastatic potential against the ovarian and breast cancer cells (Kakkar et al., 2014, Thaiparambil et al., 2011). Consistently, the migration and invasion of myeloma cells was also remarkably inhibited by withaferin A treatment in the present study. Summing up, the in vitro study results indicate the therapeutic potential of withaferin A against the human myeloma. However, same needs to be confirmed in animal systems in vivo. 


\section{CONCLUSION}

Collectively, withaferin A exhibits selective cytotoxic effects against the myeloma cells without affecting the normal plasma cell viability. The antiproliferative effects of withaferin were found to be due to its ability to induce ROS mediated intrinsic apoptosis. The study suggests the therapeutic potential of withaferin A against human myeloma. The semi-synthetic chemistry approaches might be helpful in enhancing the effectiveness of Withaferin $\mathrm{A}$ in the future.

\section{Conflict of interest}

All authors declare that there is no conflict of interest.

\section{REFERENCES}

Adham AN, Abdelfatah S, Naqishbandi AM, Mahmoud N, Efferth T (2020) Cytotoxicity of apigenin toward multiple myeloma cell lines and suppression of iNOS and COX-2 expression in STAT1transfected HEK293 cells. Phytomedicine 8: 153371. https://doi. org/10.1016/j.phymed.2020.153371

Berghe WV, Sabbe L, Kaileh M, Haegeman G, Heyninck K. Molecular insight in the multifunctional activities of Withaferin A (2012) Biochem Pharmacol 84: 1282-1291. https://doi.org/10.1016/j. bcp.2012.08.027

Cai J, Yang J, Jones D (1998) Mitochondrial control of apoptosis: the role of cytochrome c. Biochim Biophys Acta Biomembr 1366: 139-1349. https://doi.org/10.1016/s0005-2728(98)00109-1

Cragg GM, Pezzuto JM (2016) Natural products as a vital source for the discovery of cancer chemotherapeutic and chemopreventive agents. Med Princ Pract 25: 41-59. https://doi.org/10.1159/000443404

Dicato MA (2018) Multiple myeloma. In Side Effects of Medical Cancer Therapy pp 277-284. Springer, Cham

Ding X, Chen H (2018) Anticancer effects of carvone in myeloma cells is mediated through the inhibition of p38 MAPK signalling pathway, apoptosis induction and inhibition of cell invasion. JBUON 23: $747-751$

Eguchi Y, Shimizu S, Tsujimoto Y (1997) Intracellular ATP levels determine cell death fate by apoptosis or necrosis. Cancer Res 57: 1835-1840

Fulda S (2010) Modulation of apoptosis by natural products for cancer therapy. Planta Med 76: 1075-1079. https://doi. org/10.1055/s-0030-1249961

Hahm ER, Moura MB, Kelley EE, Van Houten B, Shiva S, Singh SV (2011) Withaferin A-induced apoptosis in human breast cancer cells is mediated by reactive oxygen species. PloS One 6: e23354. https:// doi.org/10.1371/journal.pone.0023354

Hassannia B, Logie E, Vandenabeele P, Berghe TV, Berghe WV (2020) Withaferin A: From ayurvedic folk medicine to preclinical anti-cancer drug. Biochem Pharmacol 173: 113602. https://doi.org/10.1016/j. bcp.2019.08.004

Iqbal J, Abbasi BA, Mahmood T, Kanwal S, Ali B, Shah SA, Khalil AT (2017) Plant-derived anticancer agents: A green anticancer approach. Asian Pac J Trop Biomed 7: 1129-1150. https://doi.org/10.1016/j.apjtb.2017.10.016

Issa ME, Cuendet M (2017) Withaferin A induces cell death and differentiation in multiple myeloma cancer stem cells. MedChem Comm 8: 112-1121. https://doi.org/10.1039/c6md00410e

Kakar SS, Parte S, Kelsey Carter IG, Worth C, Rameshwar P, Ratajczak MZ (2017) Withaferin A (WFA) inhibits tumor growth and metastasis by targeting ovarian cancer stem cells. Oncotarget 8: 74494 . https://doi.org/10.18632/oncotarget.20170

Kakar SS, Ratajczak MZ, Powell KS, Moghadamfalahi M, Miller DM, Batra SK, Singh SK (2014) Withaferin a alone and in combination with cisplatin suppresses growth and metastasis of ovarian cancer by targeting putative cancer stem cells. PloS One 9: e107596. https://doi.org/10.1371/journal.pone.0107596

Kazandjian D (2016) Multiple myeloma epidemiology and survival: A unique malignancy. In Seminars in Oncology vol 43, pp 676-681. WB Saunders. https://doi.org/10.1053/j.seminoncol.2016.11.004

Kumar SK (2019) Targeted management strategies in multiple myeloma. Cancer J 25: 59-64. https://doi.org/10.1097/ PPO.0000000000000353

Kumar SK, Rajkumar SV (2018) The multiple myelomas - current concepts in cytogenetic classification and therapy. Nat Rev Clin Oncol 15: 409-421. https://doi.org/10.1038/s41571-018-0018-y

Lee IC, Choi BY (2016) Withaferin-A - a natural anticancer agent with pleitropic mechanisms of action. Int J Mol Sci 17: 290. https://doi. org/10.3390/ijms 17030290

Mousa HA (2017) Prevention and treatment of influenza, influenza-like illness, and common cold by herbal, complementary, and natural therapies. Evid Based Complementary Altern Med 22: 166-174. https:// doi.org/10.1177/2156587216641831

Muccio VE, Saraci E, Gilestro M, Gattei V, Zucchetto A, Astolfi M, Ruggeri M, Marzanati E, Passera R, Palumbo A, Boccadoro M (2016. Multiple myeloma: New surface antigens for the characterization of plasma cells in the era of novel agents. Cytometry B Clin Cytom 90: 81-90. https://doi.org/10.1002/cyto.b.21279

Munshi NC, Avet-Loiseau H (2011) Genomics in multiple myeloma. Clin Cancer Res 17: 1234-1242. https://doi.org/10.1158/1078-0432. CCR-10-1843

Naseri MH, Mahdavi M, Davoodi J, Tackallou SH, Goudarzvand M, Neishabouri SH (2015) Up regulation of Bax and down regulation of $\mathrm{Bcl} 2$ during $3-\mathrm{NC}$ mediated apoptosis in human cancer cells. Cancer Cell Int 15: 55. https://doi.org/10.1186/s12935-015-0204-2

Pinto V, Bergantim R, Caires HR, Seca H, Guimarães JE, Vasconcelos MH (2020) Multiple myeloma: available therapies and causes of drug resistance. Cancers 12: 407. https://doi.org/10.3390/cancers12020407

Shi Y (2004) Caspase activation, inhibition, and reactivation: a mechanistic view. Protein Sci 13: 1979-1987. https://doi.org/10.1110/ ps.04789804

Singh N, Bhalla M, de Jager P, Gilca M (2011) An overview on ashwagandha: A Rasayana (Rejuvenator) of Ayurveda. Afr J Tradit Complement Altern Med 8 (5S). https://doi.org/10.4314/ajtcam.v8i5S.9

Szudy-Szczyrek A, Szczyrek M, Soroka-Wojtaszko M, Hus M (2016) New prognostic biomarkers in multiple myeloma. Postepy Hig Med Dos 70: 811. https://doi.org/10.5604/17322693.1211183

Tang Q, Ren L, Liu J, Li W, Zheng X, Wang J, Du G (2020) Withaferin A triggers $\mathrm{G} 2 / \mathrm{M}$ arrest and intrinsic apoptosis in glioblastoma cells via ATF4-ATF3-CHOP axis. Cell Prolif 53: e12706. https://doi. org $/ 10.1111 /$ cpr.12706

Thaiparambil JT, Bender L, Ganesh T, Kline E, Patel P, Liu Y, Tighiouart M, Vertino PM, Harvey RD, Garcia A, Marcus AI (2011) Withaferin A inhibits breast cancer invasion and metastasis at sub-cytotoxic doses by inducing vimentin disassembly and serine 56 phosphorylation. Int J Cancer 129: 2744-2755. https://doi. org/10.1002/ijc. 25938

Xia HZ, Gao L, Yue H, Shi BX (2018) Exploring meaning in the life of Chinese breast cancer survivors. Cancer Nurs 41: 124-130. https://doi.org/10.1097/NCC.0000000000000466

Yco LP, Mocz G, Opoku-Ansah J, Bachmann AS (2014) Withaferin A inhibits STAT3 and induces tumor cell death in neuroblastoma and multiple myeloma. Biochem Insig 7: BCI-S18863. https://doi. org/10.4137/BCI.S18863 